\title{
«Da la cuna a la tomba è un breve passo»: una lettura di due rime morali di Marino
}

\author{
Andrea Grassi \\ Università di Friburgo
}

\begin{abstract}
In queste pagine si intende rivolgere lo sguardo alle scaturigini della poesia sacra mariniana, in particolare alle Rime morali contenute delle Rime del I602. Attraverso la lettura di due tessere esemplari, si propone un'indagine che delinei alcune costanti della poesia morale del giovane Marino. Nell'analisi si contestualizzano i testi all'interno del panorama letterario dell'epoca e della tradizione classica del genere morale, mettendo in luce, oltre alla disinvoltura nel riuso e nell'intarsio delle fonti, il grado d'innovazione dell'operazione mariniana.

Keywords: Marino, Rime morali, Celio Magno, intertestualità, Ovidio
\end{abstract}

Le Rime morali rappresentano una parte quantitativamente modesta della produzione lirica mariniana: il corpus si riduce infatti a soli I6 sonetti, che assieme ai 4I sonetti delle Rime sacre, costituiscono la sezione spirituale della prima parte delle Rime pubblicate presso l'editore veneziano Giovan

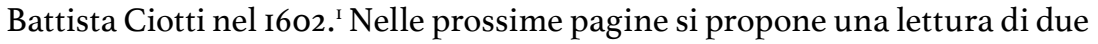
tessere attraverso le quali si cercherà di mettere in luce alcune peculiarità di questa sezione.

È bene partire da un'osservazione di Francesco Ferretti sulla produzione lirica morale dell'epoca: approfondendo l'opera di Angelo Grillo (il massimo esponente della lirica religiosa a cavallo tra i due secoli), lo studioso si è soffermato sul titolo Rime morali della prima raccolta del poeta genovese (Bergamo, 1589), individuando una distinzione tra il genere prettamente spirituale e quello encomiastico, coltivato con accenti austeri e meditativi, e per questo motivo denominato morale. ${ }^{2}$ Marino segue il modello proposto da Grillo e accoglie, nelle Rime del I602, la netta separazione tra il genere morale e quello spirituale. In seguito, quando nella Lira (I6I4) amplierà le Rime con un'inedita terza parte, questa differenziazione di genere svanirà.

I Per quanto concerne una più dettagliata contestualizzazione della lirica sacra mariniana all'intero delle Rime e della poesia religiosa del tempo, nonché degli studi specifici su questa materia, rimando ai mei contributi: Andrea Grassi, "Leggendo or tu, con miglior studio impara". Note per un attraversamento delle Rime sacre, in Marino 20I4, Atti della giornata di studi (Friburgo, 4 settembre 20I4), a cura di Sandra Clerc e Andrea Grassi, Bologna, I libri di Emil, 20I6, pp. 82-Ioo e Id., La poesia religiosa del giovane Marino: alcuni appunti sulle modalità di riuso della Bibbia nelle Rime sacre, in «Testo», LXX, 2015, pp. 4I-57, in particolare cfr. la bibliografia a p. 42 n. I.

2 Francesco Ferretti, Le Muse del Calvario. Angelo Grillo e la poesia dei benedettini cassinesi, Bologna, il Mulino, 20I2, pp. I27-I28 e n. I6. 
Nelle Rime morali mariniane è possibile ritrovare questa distinzione nelle tematiche trattate, prevalentemente ancorate alla dimensione terrena e mondana; basta dare una scorsa agli argomenti dei sonetti: da una discussione sulle «miserie umane» (n. I) e sull'«instabilità e varietà del Tempo» (n. 2), si passa alla classica «loda alla vita solitaria» (n. 5), alle invettive contro «coloro ch'edificano superbi palazzi» (n. 4) e "contro i ricercatori dell'oro» (n. I2), a un trittico su Roma (nn. 8-9-Io) che termina con la celebrazione del papato nell'anno del giubileo (n. II), per chiudere con una serie di testi di stampo encomiastico con velati richiami alla seconda carcerazione napoletana del poeta (n. I4) o alla sua adesione all'Accademia Romana (n. I6).

Il componimento che apre la sezione è tra i più celebri di Marino ed è oggetto di un episodio memorabile, a stare al racconto di una delle prime biografie sul poeta partenopeo. Infatti, nella Vita del Cavalier Marino (I633), Giovan Francesco Loredano narra che Marino

con l'occasione della stampa e con la curiosità di vedere questo mondo di meraviglie, si trasferì in Venezia, ove dalle delizie della città e dagli onori de' gentiluomini fu trattenuto lo spazio d'un anno. In questo tempo fece amicizia co 'l signor Guido Casoni cavalier, uno de' principali letterati de' nostri giorni. Gli fu mostrato in una libraria, ove era in discorso con altri virtuosi. Ambizioso di farsi conoscere, dopo il saluto recitò loro quel sonetto Apre l'uomo infelice allor che nasce e senza attender né lode né applausi di subito partì. Stupirono tutti a quella composizione, il Casoni in particolare che nella poesia ha ottenuto i primi luoghi. ${ }^{3}$

L'aneddoto, oltre a testimoniare la pratica dell'esecuzione teatrale della poesia corrente tra «virtuosi», descrive anche il primo approccio del giovane Marino, «ambizioso di farsi conoscere», con i poeti del circolo veneziano; da questa prospettiva il sonetto prende allora una forte dimensione autopromozionale. Tenendo ben presente queste dinamiche di natura sociale e poetica, possiamo procedere a una rilettura del testo:

Apre l'uomo infelice allor che nasce

in questa vita di miserie piena

pria ch'al Sol, gli occhi al pianto; e nato a pena

va prigionier fra le tenaci fasce.

Fanciullo poi, che non più latte il pasce,

sotto rigida sferza i giorni mena:

3 Giovan Francesco Loredano, Vita del Cavalier Marino, Venezia, Sarzina, I633, pp. 8-9. Sull'episodio si cfr. anche Angelo Borzelli, Il Cavalier Giovan Battista Marino (1569-I625), Napoli, Gennaro M. Priore, I898, pp. 53-54 e Id., Storia della vita e delle opere di Giovan Battista Marino, Napoli, Tipografia degli Artigianelli, 1927, pp. 63-64. 
indi in età più ferma e più serena

tra Fortuna et Amor more e rinasce.

Quante poscia sostien tristo e mendico

fatiche e morti, infin che curvo e lasso

appoggia a debil legno il fianco antico?

Chiude alfin le sue spoglie angusto sasso

ratto così che sospirando io dico:

da la cuna a la tomba è un breve passo. ${ }^{4}$

Il sonetto s'innerva su un'ingegnosa struttura lineare: il poeta ripercorre l'intera parabola umana attuando una distribuzione simmetrica dei motivi di infelicità nelle varie età dell'uomo. I periodi dell'esistenza sono scanditi da una filigrana di parole-chiave: «pianto» (v. 3), «fasce» (v. 4), «rigida sferza» (v. 6), «Fortuna e Amor» (v. 8), «fatiche e morti» (v. IO), «debil legno» (v. II) e infine «spoglie» (v. I2). Il «pianto» e le «fasce» son inseriti nella quartina dedicata alla nascita; la «rigida sferza» del periodo scolastico e il classico binomio «Fortuna e Amor», tradizionalmente propri della giovinezza, figurano nella seconda quartina, ad essa dedicata; «fatiche e morti» e il «debil legno» rientrano nella prima terzina, che tratta del passaggio dalla maturità alla vecchiaia; per terminare con la «tomba» che sigilla il sonetto e anche la parabola della miserrima vita dell'uomo.

Si sottolineano inoltre i verbi «Apre» (v. I) e «Chiude» (v. I2) che circoscrivono l'intera parabola esistenziale narrata nel sonetto e la climax ascendente che ne cadenza l'andamento: dalla nascita alla vecchiaia si riscontra un progressivo incremento di tristezza e d'infelicità, fino al momento della morte. La sentenza finale, vero e proprio fulmen in clausula introdotto dallo stesso poeta («io dico» v. 13), condensa epigrammaticamente il motivo della fugacità della vita. Si noti anche che l'unica interrogativa del testo, adagiata nella prima terzina, risulta squisitamente retorica: non vi è infatti alleviamento alle pene nella vita terrena. Proprio questa terzina, dedicata alla maturità e alla vecchiaia, intensifica l'atmosfera di disillusione che ammanta il componimento grazie a un tessuto impreziosito da una forte bimembrazione: l'armonico andamento binario delle tre coppie che innervano i versi 9-Io viene sigillato dal chiasmo «debil legno» - «fianco antico».

4 Si è utilizzato l'esemplare della princeps conservato nel fondo Giovanni Pozzi presso la Biblioteca Salita dei Frati di Lugano: Giovan Battista Marino, Rime, Venezia, Ciotti, 1602. Per la descrizione della secentina si rinvia alla scheda num. 90 di Francesco Giambonini, Bibliografia delle opere a stampa di Giambattista Marino, Firenze, Olschki, 200o. Oltre alla princeps si è usufruito, in costante confronto, delle recenti edizioni della Lira curate da Slawinski (Giovan Battista Marino, La Lira, a cura di Maurizio Slawinski, Torino, RES, 2007) e da Salvarani (Giambattista Marino, La Lira I6I4, a cura di Laura Salvarani, Lavis, La Finestra Editrice, 20I2). 
Il sonetto presenta un'interessantissima filigrana intertestuale. Edoardo Taddeo ha messo in luce il rapporto tra la poesia mariniana e la terza stanza della canzone Deus del poeta veneziano Celio Magno. ${ }^{5}$ Nella canzone, che ebbe un notevole successo alla fine del ' 500 , tanto da essere stata edita nel I597 corredata dai copiosi commenti di Ottavio Menini, Valerio Marcellini e Teodoro Angelucci, ${ }^{6}$ il poeta, seduto sulla riva del Giordano, vede scendere dal cielo delle dee, una delle quali prende la parola e gli illustra la caducità della vita umana, ammonendolo della futilità delle cose terrene e ricordandogli che il fine ultimo dell'uomo è l'ascesa al paradiso, il ritorno a Dio. Si riportano alcuni versi delle stanze 3,4 e 5 che sintetizzano anche il traliccio ideologico della canzone (vv. 4I-9I):

Apre nascendo l'uom pria quasi al pianto stanza 3 ch'a l'aria gli occhi: e ben quinci predice gravi tormenti a' suoi futuri giorni.

Né qua giù vive altro animal che tanto sia di cibo e vestir privo e infelice; né che in corpo più fral di lui soggiorni. L'accoglie poi tra mille insidie e scorni il mondo iniquo, e'n labirinto eterno di travagli e d'error l'intrica e gira:

[...]

Ti vedrai giunto al duro ultimo passo, stanza 4 e gli amici più cari e i dolci eredi con ogni tuo desir lassando a dietro, fredda esangue n'andrai soma in feretro.

[...]

Ma questo divin lume in voi ridutto stanza 5 giamai non more: in voi l'anima regna, che del corporeo vel si veste e spoglia; laqual, s'ogni sua voglia sprona a virtù, del ciel si rende degna. ${ }^{7}$

5 Edoardo Taddeo, Celio Magno e Marino, in Id., Studi sul Marino, Firenze, Edizioni Remo Sandron, I97I, pp. I33-I43. Sulla figura di Magno si rimanda a Daniele Ghirlanda, Celio Magno, in $D B I$, vol. LXVII, 20o6, pp. 496-498 e alla relativa bibliografia.

6 Deus Canzone Spirituale di Celio Magno. Con un Discorso sopra di quella dell'Eccellentissimo Signor Ottavio Menini. Un commento dell'Eccellentissimo Signor Valerio Marcellini, e Due lettioni dell'Eccellentissimo Signor Theodoro Angelucci, Venezia, Domenico Farri, 1597. Per la contestualizzazione e l'approfondimento di questi commenti si rimanda al bel saggio di Giuseppina Stella Galbiati, Contributo per Celio Magno: una lettura della canzone Deus, insieme ai suoi antichi commentatori, in Studi di onomastica e letteratura offerti a Bruno Porcelli, Pisa-Roma, Gruppo Editoriale Internazionale, 2007, pp. I29-I44.

7 Celio Magno, Deus, in Id., Rime, Venezia, Muschio, I6oo, pp. I27-I33, vv. 4I-9I. Non si riscontrano varianti nel brano rispetto alla versione edita nel 1597 . 
Nell'incipit del sonetto Marino riprende la frase iniziale della stanza di Magno («Apre... occhi»), ${ }^{8}$ riutilizzando, come ha ben osservato Taddeo, l'«effetto principale (Apre ad inizio di verso, tonalità grave accentuata dall'immediata inserzione di infelice, che il Magno impiega al v. 5) e la struttura ingegnosa, nella quale si realizza un miglioramento tecnico (gli occhi non si aprono propriamente "a l'aria", ma alla luce, "al sol")».9 Il «miglioramento tecnico» di cui parla Taddeo potrebbe riallacciarsi direttamente alla lettura del commento di Valerio Marcellini, il quale chiosa ampiamente i primi versi della terza stanza, scomodando addirittura la figura di Adamo e il peccato originale:

\begin{abstract}
Non aprì gli occhi prima al pianto il primo uomo, ma ai diletti del Paradiso quando da prima lo fece Iddio. Il peccato, nel quale egli cadde fu quello (dice la scrittura sacra) che il colmò di tutti quei mali di questa misera vita umana ove fu mandato a patire, così piena e colma si vede. Perciò che avendo il primo nostro padre cangiato in lagrime i suoi diletti dopo il peccato, vivendo il rimanente della sua vita in continue fatiche, in pianto; lasciò per eredità a' figliuoli, che prima aprissero gli occhi al pianto, che a questa luce del sole: per dimostrare ch'egli nasceva per dover vivere penosa e misera vita; e 'l recarsi a mente i nostri guai, che nei primi versi di questa terza stanza il Poeta ci mette innanzi per farci ricordare del peccato che ci ha cagionati. ${ }^{\circ}$
\end{abstract}

Nelle altre parti del sonetto Marino si distacca dal modello sostituendo alle considerazioni di natura morale connesse alla malvagità del mondo, una costruzione che punta sulla suggestiva scansione dell'esistenza umana all'insegna della vanità. Magno inoltre pone tutta l'esistenza dell'uomo, dalla nascita alla morte, sotto il segno del dolore, tuttavia, come si può notare dai versi proposti della quinta stanza, il poeta veneto risolve la questione in chiave escatologica contrapponendo («Ma») l'infelicità terrena alla gloria divina.

Alla canzone di Magno vanno aggiunte altre due tessere che Marino sicuramente conosceva: anzitutto, come ha ben ravvisato Benedetto Croce, ${ }^{\text {II }}$ il sonetto di Camillo Pellegrino Dura condizion de l'uom che nasce, in secondo luogo, il testo Breve di verno e nubiloso giorno di Angelo Grillo. Il primo si tro-

8 Vania de Maldé ha suggerito come possibile fonte anche dei versi del Mondo creato di Tasso: «È nato a pena il fanciulleto ignudo, / Che si riguarda il sesso; e poi s'aspetta / Il pianto, segno de l'umana vita / lacrimoso e dolente, a lei conforme» (II, 685-688). Cfr. Antologia della poesia italiana, diretta da Cesare Segre e Carlo Ossola, vol. II, Quattrocento-Settecento, Einaudi-Gallimard, Torino, I997, p. 910.

9 Taddeo, Celio Magno e Marino, cit., p. 137.

Io Magno, Deus Canzone Spirituale, cit., p. $39 \mathrm{r}$ e v. Il corsivo è mio.

II Benedetto Croce, Poeti e Scrittori del pieno e del tardo Rinascimento, vol. III, Bari, Laterza, I952, pp. 309-310. 
va nella raccolta Rime edite a Firenze nel 1584 assieme a quelle degli amici Benedetto Dell'Uva e Giambattista Attendolo.

Dura condizion de l'uom che nasce ignudo e piange nel veder la luce; e quasi anzi pur reo si lega in fasce e come bruto il senso ha sol per duce.

Indi, s'a l'età ferma si conduce, di varie cure e passioni il pasce il lume di ragion, che 'n lui riluce, mentre vuol che quel segua e questo lasce.

Ira, sdegno, desio, speranza e tema son gli avoltoi che rodon sempre il core di Tizio e Ixion la rota ultrice.

Da morbi afflitto ne l'etate estrema, la morte ha presso, e non vive né more. Vita del miser uom, dubbia, infelice! $!^{12}$

Il secondo invece è il sonetto d'apertura delle Pompe di Morte, la sezione lugubre delle Rime di Angelo Grillo nell'edizione del I599. La poesia è dedicata a Beatrice Fibbia, per la morte del figlio Alessandro.

Breve di verno e nubiloso giorno è questa mortal vita; e da che uom nasce morir comincia; e son le prime fasce, e la cuna feretro al gran ritorno.

E lunga vita mai più lungo scorno non fu, da che fu vita. Ahi, che rinasce sempre il mondo peggiore e sol si pasce di ciò ch'ancide e se ne rende adorno.

E di lagrime il duolo ingiusto oltraggio sono a chi fugge a tempo, e di quel puote a ragion dirsi: ei venne e vide e vinse.

Così stim'io del giovinetto e saggio vostro Alessandro, che partendo strinse l'armi ond'eterno è su l'empiree rote. ${ }^{13}$

I2 Rime di Benedetto Dell'Uva, Giovanbattista Attendolo, et Camillo Pellegrino, Firenze, Sermartelli, I584, p. IO7.

I3 Angelo Grillo, Rime, Venezia, Ciotti, 1599, p. 197. 
Marino, al solito, amalgama e rielabora i vari materiali: la catena rimica nasce: fasce: rinasce: pasce sembra ripresa dal sonetto di Grillo, così come la suggestiva immagine che sovrappone la culla al feretro (e si noti come l'ossimorico accostamento «cuna feretro» di Grillo sia ben più ardito di quello mariniano) che innerva la sentenza finale della prima morale: «da la cuna a la tomba è un breve passo» (con soluzione più piana, ma più efficace). ${ }^{14}$ Da Pellegrino, amico del poeta nel suo periodo giovanile, ${ }^{15}$ invece Marino riprende l'idea della disposizione per epoche della vita nella quadratura strofica del sonetto: Pellegrino, dopo aver enumerato la nascita (vv. I-4), l'età matura («età ferma» v. 5), si arresta all'«etate estrema» (v. I2), mentre Marino, oltre a dedicare particolare attenzione alla vecchiaia (vv. 9-II), chiude il cerchio con l'«angusto sasso» (v. I2). Inoltre il quarto verso del sonetto mariniano («va prigionier fra le tenaci fasce») palesa una parentela con il «reo si lega in fasce» (v. 3) di Pellegrino. Si noti anche la somiglianza lessicale nella descrizione dell'età matura: Marino ricalca sia l'avverbio («indi») sia l'aggettivo («ferma»). Proprio il sintagma «età più ferma» è oggetto, come ha ben notato Salvarani, di un'interessante variante: nell'edizione della Lira del I6I4 in luogo di «indi in età più ferma e più serena», il poeta ha scritto «indi in età più fosca, che serena». La studiosa giustifica la variante affermando che essa si allineerebbe alla pratica del tardo Marino «di eliminare le dittologie, il cui insipido andamento binario è così tipico del sonetto petrarchista». ${ }^{16}$ Una tendenza senz'altro interessante, ma che andrebbe confermata da un più largo spoglio. Nel caso specifico le ragioni del ritocco sembrano

I4 L'immagine affonda le sue radici nella tradizione cristiana, si rimanda alla voce cuna ne Il mondo simbolico dell'abate Picinelli: «Alla cuna, per titolo d'Emblema morale, può soprascriversi: ARRHA SEPULCHRI; ben sapendosi, che chiunque nasce, è necessitato a morire, e come cantò il Marino: "Da la cuna alla tomba è un brieve [sic] passo". Nel tomo 8 di S. Girolamo, nell'Ep. 6 ad amicum grotum, facendosi riflessione all'Oracolo della sapienza 7.6 "Unus introitus ad vitam, et similis exitus", così soggiunge: "O angustum, et satis anxium tempus vitae mortalis, ubi cum initio nominatur et finis. Initium nostrum cum fine suscipimus". Tertulliano dice che le fasce onde si ristringono i fanciulli sono preludi dei lenzuoli onde s'avvolgono i defonti: "Involvitur infans pannis, velut sepulturae involucris initiatus". E con alto mistero, i Santi Magi portarono alle cune di Cristo la mirra, che suol servire a curar i corpi dei defonti: come ricordare che la cuna altro non sia che una caparra espressa del catalletto e della tomba; ben dicendo Manilio: "Nascentes morimur, finisque ab origine pendet" [Astronomicom IV I6]» (Filippo Picinelli, Il mondo simbolico, Milano, nella stampa di Francesco Vigone, I68o, lib. XV, cap. IX, p. 6I9). Si noti, in particolare, la somiglianza tra la sentenza di Tertulliano e quella di Marino.

I5 I due si conobbero nel 1596 per intercessione di Alessandro Pera (ne scaturì lo scambio poetico poi pubblicato nelle Proposte et Risposte delle Rime mariniane) e si rincontrarono nel marzo del 1598 presso la casa del Marchese di Campolattaro, occasione in cui il Pellegrino ideò l'opera Del Concetto poetico. Il dialogo tratta della poesia lirica della fine del Cinquecento e al suo interno il personaggio di Marino si fa portavoce delle nuove tendenze poetiche. Cfr.

Grassi, La poesia religiosa..., cit., pp. 50-5I e n. 3.

I6 Marino, La Lira I6I4, cit., p. 37I. 
altre. Marino, in questo testo, non voleva eliminare le dittologie: mal si spiegherebbe perché la prima terzina non viene toccata, nonostante densità di bimembrazioni (si tratta inoltre della terzina in cui vi è un palese richiamo a $R v f$ I4). ${ }^{17}$ Piuttosto si direbbe che la variante sia stata indotta da motivi semantici ed esistenziali; che vi sia, a monte, un cambiamento di prospettiva: a distanza di una decina d'anni il poeta non vede più la maturità come un'età «ferma e serena», ma significativamente «fosca». La variante prende ulteriore profondità alla luce dell'intertestualità pellegriniana: è probabile infatti che il giovane Marino si sia avvalso del sintagma del sonetto dell'amico più anziano per descrivere l'età matura; in seguito, ad anni di distanza, inasprisce la rappresentazione di questo periodo della vita, conferendo al testo un maggior grado di disillusione..$^{18}$

In questo sonetto Marino ha rielaborato i testi di tre poeti coi quali ha avuto (come nel caso di Pellegrino) o stava instaurando proprio in quel periodo (come nel caso di Grillo e Celio Magno) dei rapporti di amicizia che implicavano scambi di materiali epistolari e poetici; e dei quali conosceva bene le opere pubblicate. Se confrontiamo il sonetto di Marino con gli altri testi emerge un dato molto interessante: Magno e Grillo risolvono i loro componimenti in chiave escatologica. L'uscita, quindi, è sempre, nonostante tutto, di valore positivo: mentre Pellegrino si ferma alla miseria della vita inclusa tra nascita e vecchiaia (non viene però contemplata la tomba e la morte), Marino invece percorre tutta la parabola esistenziale sigillando il componimento con una lapidaria sentenza, senza tuttavia accennare a una soluzione positiva che possa riscattare l'esistenza umana. Marino resta significativamente reticente, forse per rimarcare ancor di più quella distinzione di campi già palesata dalla separazione tra le Rime sacre e le Rime morali.

Torniamo infine all'episodio raccontato da Loredano: alla luce di quanto visto finora, la scelta da parte del poeta di Apre l'uom infelice allor che nasce per promuovere la sua poesia nei circoli veneziani pare emblematica. Guido Casoni, soprattutto grazie alla sua raccolta di odi - peraltro ripubblicata presso il Ciotti qualche mese prima dell'arrivo di Marino a Venezia -, va considerato, come indicano gli studi di Besomi e di Corradini, ${ }^{19}$ un «perso-

I7 «indi trahendo poi l'antiquo fianco / per l'extreme giornate di sua vita, / quanto più po', col buon voler s'aita, / rotto dagli anni, et dal camino stanco» Rvf I4, 5-8 (rime: biancho: manco: fianco: stanco). È inoltre probabile l'incrocio con alcuni versi del Rinaldo di Tasso (Rinaldo I 3I): «Huom riscontrò d'aspetto venerando, / di crespe rughe e il volto ingombro, e pieno / che sovra un bastoncel giva appoggiando / le membra che parean venir già meno». I corsivi sono i mei. I8 Inoltre l'aggettivo variato crea un collegamento con il decimo verso del terzo sonetto della sezione: «dentro l'abisso luminoso e fosco».

I9 Per il rapporto tra Marino e Casoni si rimanda anche a Ottavio Besomi, Ricerche intorno alla Lira, Padova, Antenore, 1969, pp. I3I-54 e a Marco Corradini, La ricerca metaforica di Guido Casoni, in Id., La tradizione e l'ingegno. Ariosto, Tasso, Marino e dintorni, Novara, Interlinea, 2004, pp. 95-II2. 
naggio di primo piano nella storia della lirica del '600», ${ }^{20}$ sicuramente tra gli esponenti più in vista del circolo veneziano; inoltre l'opera del poeta veneto ebbe grande influenza su quella di Marino (a questo proposito si ricordi almeno il caso dell'ode $O$ bellissima Clori, nella quale si svolge il tema della rosa, ripreso e reso celeberrimo da Marino prima nella Canzona della rosa e poi in Adone III 66-67); ${ }^{21}$ infine va ricordato che Casoni fu anche poeta morale e in particolare si segnala la fortissima prossimità tematica tra il sonetto e l'ode Poca polva animata dedicata a Giacomo Zane che tratta, come indicato dall'argomento, «la miseria dell'uomo»; sebbene l'ode non palesi particolari connessioni sintagmatiche con il sonetto, sin dalla prima strofa si presenta pertinente al tema svolto da Marino. ${ }^{22}$ Il sonetto colpì nel segno e tra l'esordiente poeta partenopeo e Casoni nacque un'amicizia.

Tuttavia la portata autopromozionale del componimento non si arresta al solo Casoni, bensì va estesa, come dimostra l'analisi dell'intertestualità, anche a Celio Magno; autore di primo piano nella Venezia della fine del '500: aveva appena dato alle stampe, assieme all'amico Orsatto Giustiniano, il lavoro poetico di una vita, le Rime edite a Venezia nel I6oo. Egli fu inoltre una figura molto importante per l'esordio poetico del primo Marino: infatti, oltre a incontrare il giovane poeta durante il soggiorno veneziano, ebbe con lui ben due scambi poetici (unico caso nelle corrispondenze poetiche mariniane) e mise la sua firma all'edizione delle Rime in calce all'approvazione dei riformatori dello Studio di Padova (all'epoca lo scrittore era segretario del Consiglio dei Dieci). Non è quindi improbabile che Marino, presentandosi nei circoli veneziani, abbia deciso di usare proprio una delle tessere che più omaggiano il poeta veneziano. Al solito però l'omaggio diventa in Marino occasione di gareggiamento, al tempo stesso di incontro e di superamento del modello.

Ritroviamo questo approccio anche nei confronti della tradizione latina di ambito morale. Il dodicesimo sonetto della sezione ne è una tessera esemplare; si tratta di un'invettiva, come esplicitato dalla didascalia, «Contro i ritrovatori dell'Oro».

Pera chi pria da le secrete e basse vene de' monti, o dal Tartareo fondo

20 Besomi, Ricerche intorno alla Lira, cit., p. I54.

2I Ibidem, pp. I36-I37.

22 Si riportano la prima e le ultime due strofe dell'ode: «Poca polva animata, / uomo più tosto morto che mortale, / questa tua vita amata, / fungo notturno e frale, / svanisce, e questo mondo / che si vagheggi in sua bellezza immondo. [...] Senza figli ti duole / essere ignudo tronco, e se diventi / padre di molta prole / provi mille tormenti, / sfrenato e troppo ardente / giovane, e vecchio se' freddo e languente. / Quel bambino lattante / piagne, e rider non sa, forse lo sdegna. / O ben dotto ignorante, / che'a te superbo insegna, / come pianger nascendo / sol deve l'uomo, e poi morir ridendo». Si cfr. Guido Casoni, Ode, Venezia, Ciotti, I6oI, pp. II5-II6. 
sprigionò l'oro scelerato immondo, e chi trattollo, e chi l'accolse in masse.

Seco l'inganno allor, seco allor trasse la morte, e 'l morbo universal del mondo, che di Saturno il secolo giocondo lieto menò, quantunque ignudo errasse.

Ebbe di ferro il cor chi da l'ascose viscere de la terra il ferro tolse, ma nemico men fero almen n'espose.

Quegli i corpi a ferir l'ingegno volse, questi dal chiuso, in cui Natura il pose, l'omicida de l'anime disciolse.

Con questa l'invettiva Marino affronta un topos caro alla tradizione della poesia sacra e morale, sovente rielaborato attraverso la famosa matrice virgiliana rifacentesi al terzo canto dell'Eneide (III 56-57). ${ }^{23}$ Il poeta rimane ancorato ai classici, si appoggia tuttavia agli amati elegiaci, intarsiando la lezione di Tibullo con quella di Ovidio. La prima traccia, già suggerita da Menghini ${ }^{24}$, si riscontra nell'incipit della decima elegia del primo libro di Tibullo (Eleg. I Io, I-8):

Quis fuit, horrendos primus qui protulit enses?

Quam ferus et vere ferreus ille fuit!

Tum caedes hominum generi, tum proelia nata, tum brevior dirae mortis aperta via est.

An nihil ille miser meruit, nos ad mala nostra vertimus, in saevas quod dedit ille feras?

Divitis hoc vitium est auri, nec bella fuerunt, faginus adstabat cum scyphus ante dapes. ${ }^{25}$

Alla quale vanno affiancati i versi relativi all'età del ferro del primo libro delle Metamorfosi ovidiane (I I25-I44):

Tertia post illam successit aënea proles, saevior ingeniis et ad horrida promptior arma,

23 Salvatore Ussia, Il Sacro e il Parnaso. Il Lauro e la Croce, Catanzaro, Pullano Editori, I993, p. 42.

24 Mario Menghini, La vita e le opere di G. B. Marino, Roma, I888, pp. 75-76.

25 Per i testi latini di seguito citati si sono utilizzate le seguenti edizioni: Albio Tibullo, Elegie, a cura di Annalisa Németi, Milano, Mondadori, 2006; Publio Ovidio Nasone, Metamorfosi, a cura di Piero Bernardini Marzolla, Torino, Einaudi, 1994; Publio Ovidio Nasone, Amori, a cura di Ferruccio Bertini, Milano, Garzanti, 2003. I corsivi nel testo sono i miei. 
non scelerata tamen. De duro est ultima ferro.

Protinus inrupit venae peioris in aevum

omne nefas, fugitque pudor verumque fidesque;

in quorum subiere locum fraudesque dolique

insidiaeque et vis et amor sceleratus habendi.

[...]

Nec tantum segetes alimentaque debita dives

poscebatur humus, sed itum est in viscera terrae, quasque recondiderat Stygiisque admoverat umbris,

effodiuntur opes, inritamenta malorum.

Iamque nocens ferrum ferroque nocentius aurum

prodierat: prodit bellum, quod pugnat utroque,

sanguineaque manu crepitantia concutit arma.

Se dall'elegia di Tibullo Marino trae in particolare la paronomasia «fero» - «ferro» della seconda terzina, è tuttavia l'ipotesto ovidiano a fungere da vero e proprio canovaccio concettuale del sonetto: dall'ubicazione sotterranea e infernale del metallo (vv. I-4), alla matrice delle tessere lessicali «scelerato» (v. 3), «immondo» (v. 3) e «inganno» (v. 5), nonché alla contrapposizione tra il ferro e l'oro, con la palma del metallo più nefasto consegnata a quest'ultimo (vv. 9-I4). A queste due tessere, incrociate con la tipica disinvoltura mariniana, è necessario ricordare un'ulteriore filigrana ovidiana: $\mathrm{i}$ versi 35-54 dell'ottava elegia del terzo libro degli Amores. Dopo aver denunciato la sostanziale inutilità della poesia amorosa per conquistare l'amata, e accennato alla vittoria del suo rivale in amore ( Ecce, recens dives, parto per vulnera censu, / praefertus nobis sanguine pastus eques» III, 8, 9-IO), Ovidio recupera il motivo topico dei Saturnia regna, sfruttato per introdurre una dura requisitoria contro la società augustea che appare dominata dalla brama di denaro e di ricchezze, conquistate versando sangue.

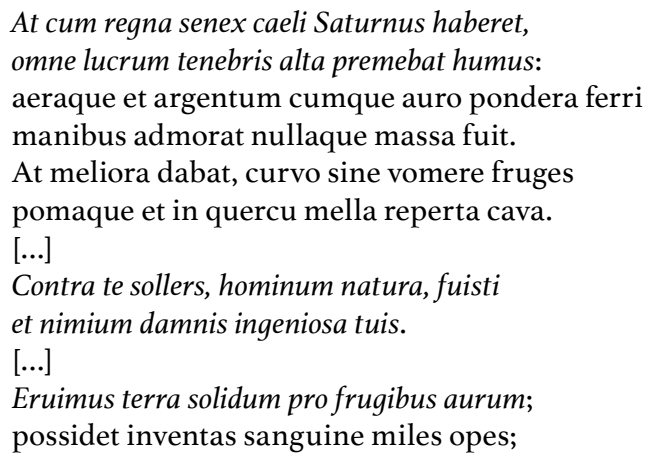

La tessera elegiaca è reperto prezioso, oltre che per l'allusione alla topica età dell'oro e al suo re Saturno, paradossalmente nominata con il metallo 
che ne sancisce la fine, soprattutto per quella «massa» che poi nel sonetto mariniano si staglia in rima, costringendo le vene dei monti a divenire non solo, al solito, «secrete», ma anche «basse» (v. I), che pare elemento nuovo.

Rispetto ai modelli latini, Marino declina il tema sotto una decisa connotazione religiosa che ne riorienta l'interpretazione, rinnovandolo non solo nell'elocutio. A questo proposito è fondamentale la chiusa: infatti il poeta risolve il confronto tra i due metalli nella classica dicotomia tra il corpo e l'anima, corroborata altresì nell'intensità semantica che connota i due materiali: si passa dal «ferir» (v. I2) legato al ferro al più marcato «omicida» (v. I4) applicato direttamente all'oro. ${ }^{26}$

Anche il lavoro sulle componenti strutturali e formali del testo è accurato e funzionale. L'intero testo si organizza attorno al movimento binario: dalla classica bimembrazione, alla ripresa delle particelle che segnano il procedere logico e speculativo del sonetto «chi... chi» (vv. I-4), «Seco... seco» (vv. 5-8), «Quegli... Questi» (vv. 9-II). La prima terzina segna uno scarto inserendo un'avversativa, che rimarca anche il passaggio da un orientamento orizzontale a quello verticale di matrice anaforica evidenziato dai deittici dei versi I2-I3. Oltre all'attacco sostenuto, e del tutto inedito in questa sezione morale, che sviluppa l'assunto di matrice ovidiana, è particolarmente efficace la saldatura tra i due movimenti del sonetto, rafforzata dalle due forti inarcature che aprono la fronte (vv. I-2) e la sirma (vv. 9-Io).

Il componimento è inoltre disseminato da un'articolata trama di movimenti iterativi e allitterativi, in cui spiccano due filigrane che perpetuano i metalli protagonisti del sonetto: l'oro riverbera in «allOR... allOR» (v. 5); «mORte... mORbo» (v. 6); «cOR» (v. 9); «cORpo» (v. I2); mentre il ferro si ripercuote nel trinomio su vibrante geminata «fERRo» - «tERRa» - «fERRo» dei versi 9 e IO, preannunciato dall'«ERRasse» (v. 8), e, ai versi II e I2, nell'annominatio con «fero»e «ferir».

Da questa prima indagine emerge un Marino attento a distinguersi rispetto al panorama letterario che lo circonda, capace di rinnovare i topoi applicando nuove logiche e di muoversi con disinvoltura all'interno del genere creando un discorso poetico che tende a rompere la continuità con il modello petrarchista. Anche per le morali si conferma l'intuizione di Alessandro Martini che ritrova nella lirica mariniana

26 A complemento dei modelli latini, sicuramente preminenti nella memoria del poeta napoletano, si riporta anche la fronte del sonetto 7I delle Rime spirituali di Gabriele Fiamma: «Qual uom, che, intento a cercare gemme et oro, / apre a l'antica madre il petto e 'l seno, / e d'ingorde speranze accesso e pieno / d'or in or di trovar crede il tesoro; / ma quanto più profonda il suo lavoro, / tanto men ricco ogni or scopre il terreno: / onde al fin e il suo error conosce a pieno / e 'ndarno cerca a' suoi danni ristoro» (Gabriele Fiamma, Rime spirituali, Venezia, Francesco de' Franceschi, I570, son. LXXII, p. 253, vv. I-8). Va ricordato anche che l'intero testo si struttura su una similitudine con figurante nelle quartine relativo al ricercatore dell'oro. 
la rinuncia a modelli totalizzanti a favore di un più libero e magari effimero gioco letterario, esaltante l'autonomia del linguaggio poetico, l'assidua pratica ludica di un'arte chiusa in se stessa, che non presume di cambiare il mondo, il prelievo e la giustapposizione di elementi stilistici delle più svariate tradizioni, senza pretese di continuità storica. ${ }^{27}$

andrea.grassi2@unifr.ch

27 Alessandro Martini, Marino postpetrarchista, in «Versants», VII, I985, pp. 33-34. 